\title{
Embarazo no deseado en adolescentes, y utilización de métodos anticonceptivos posparto
}

\author{
Rosa María N úñez-U rquiza, MC, MSP,(1) Bernardo Hernández-Prado, PhD, ${ }^{(1)}$ C ecilia García-Barrios, MC, (2) \\ Dolores González, MSP, (1) Dylis W alker, MC. (1)
}

\section{Núñez-Urquiza RM, Hernández-Prado B, García-Barrios C, González D, Walker D. Embarazo no deseado en adolescentes, y utilización de métodos anticonceptivos posparto. Salud Publica Mex 2003;45 supl 1:S92-S102.}

\section{Resumen}

Objetivo. D escribir la proporción de embarazo no deseado en adolescentes, su asociación con características sociodemográficas y la utilización de anticonceptivos posparto. Material y métodos Se trata de un estudio transversal con una muestra de 220 mujeres adolescentes entre 13 y 19 años de edad, de dos municipios semiurbanos del estado de Morelos, México, entrevistadas entre 1992 y 1994. Las mujeres fueron entrevistadas en su casa entre seis y $12 \mathrm{se}-$ manas después del parto. Se les preguntó si antes de embarazarse desear on el último embarazo.A demás, se indagó sobre conocimientos y uso de métodos anticonceptivos posparto. Resultados Un $17 \%$ de todos los nacimientos registrados en la población total en ese perio do fueron producto de madres adolescentes. De éstas, $22.73 \%$ declararon que su embarazo no fue deseado. Se encontró una asociación positiva entre no tener derecho de acceso a los servicios de salud de las instituciones del sistema de seguridad social - Instituto Mexicano del Seguro Social (IMSS), Instituto de Seguridad y Servicios Sociales de losTrabajadores del Estado (ISSSTE) - y embarazo no deseado (RM ajustada=3.03, IC 95\% 1.31, 7). Asimismo, las adolescentes de comunidades urbanas manifestaron no deseo del embarazo con mayor frecuencia que las mujeres de comunidades rurales (RM ajustada=2.16, IC 95\% 1.08, 4.33). Un 91.3\% de las madres adolescentes enunció la píldora anticonceptiva entre los méto dos que conocía. Un $84.72 \%$ conocía el DIU y $63.68 \%$ el condón. Sin embargo, sólo 35\% estaba utilizan-

\author{
Núñez-Urquiza RM, Hernández-Prado $B$, \\ García-Barrios C, González D, Walker D. \\ Unwanted adolescent pregnancy \\ and post-partum utilization of contraceptive methods. \\ Salud Publica Mex 2003;45 suppl 1:S92-S102.
}

\begin{abstract}
A bstract
Objective. To describe the proportion of unwanted pregnancies among all pregnant adolescents, its association with sociodemographic characteristics, and the use of postpartum contraceptive methods. Material and Methods A cross-sectional study was conducted among 220 women between 13 and 19 years of age, in two semi-urban municipalities of the State of Morelos, Mexico, inter viewed between 1992 and 1994.W omen were inter viewed at home, six to twelve weeks after their delivery date.W omen were asked whether they had wanted their last pregnancy, and about knowledge and use of contraceptive methods after delivery. ResultsAdolescent pregnancies accounted for $17 \%$ of all births registered in these two municipalities. Among all adolescent mother $22.73 \%$ reported that their pregnancy had not been wanted. A positive association was found between the lack of access to health services provided by public medical insurance systems (Instituto Mexicano del Seguro Social IMSS and Instituto de Seguridad y Servicios So ciales para los Trabajadores del Estado ISSSTE) and unwanted pregnancy (adjusted O R=3.03, 95\% Cl $(1.31,7$. An association was also found between living in an urban community (adjusted $\mathrm{OR}=2.16,95 \% \mathrm{Cl}(1.08,4.33)$ and an unwanted pregnancy. Among all adolescent mothers, $91.3 \%$ were familiar with "the pill" as a contraceptive method; $84.72 \%$ knew about the IUD, and $63.68 \%$ knew about the condom. However, only $35 \%$ of them were actually using an effective contraceptive method six weeks after delivery. N 0
\end{abstract}

La Fundación John \& Catherine McActhur proporcionó apoyo financiero para este proyecto.

(1) Instituto Nacional de Salud Pública, Cuernavaca, Morelos, México.

(2) Secretaría de Salud de México, México, D.F.

Fecha de recibido: 18 de abril de 2001 - Fecha de aprobado: 3 de junio de 2002

Solicitud de sobretiros: Rosa María N úñez-U rquiza. Avenida Universidad 665. Cuernavaca Morelos, México 62508.

Correo electrónico: rmanunez@ correo.insp.mx 
do algún método anticonceptivo efectivo después de las seis semanas posparto. No se encontró diferencia en la frecuencia de uso de anticonceptivos según deseo del último embarazo. Entre quienes se atendieron el parto en centros de salud u hospital sólo 43.39\% estaba utilizando algún método efectivo de planificación familiar. Conclusiones Estos hallazgos sugieren que los programas de planificación familiar tienen un potencial de cobertura aún no alcanzado entre el grupo de adolescentes, especialmente entre las que viven en áreas suburbanas y entre las no derechohabientes de la seguridad social. A simismo, sugiere la necesidad de insistir en la promoción de la utilización de métodos anticonceptivos posparto en este grupo considerado de alto riesgo reproductivo. Además, se evidencia la necesidad de investigar sobre métodos de educación sexual y reproductiva que puedan introducirse en el sistema escolar desde el nivel primario.

Palabras clave: embarazo no deseado; embarazo en adolescencia; planificación familiar; México difference in frequency of contraceptive use was found among the adolescent mothers, according to whether they wanted their last pregnancy. 0 nly $43.39 \%$ of mothers who delivered at hospitals or health centers were using an effective contraceptive method. ConclusionsThese findings suggest that there is a great potential for family planning programs to target adolescents, and that the use of contraceptive methods after delivery should be promoted among adolescent mothers, especially those lacking access to public medical insurance and those living in semi-urban settings. It is also recommended to conduct studies on family planning education programs that can be introduced earlier in the school system in Mexico.

Key words: unwanted pregnancy; pregnancy in adolescence; family planning; Mexico
L a salud sexual y reproductiva de la población adolescente se ha considerado, en México, como una prioridad de atención dentro del Plan Nacional de Salud 1995-2000. ${ }^{1}$ En México, la tasa de fecundidad global descendió entre 1970 y el año 2000 de 6.0 a tres hijos por mujer. ${ }^{2}$ Sin embargo, debido a la elevada proporción de población joven, la cantidad de mujeres en edad reproductiva se duplicó en el mismo periodo. Así, aunque la tasa de fecundidad en la población ha disminuido, la cantidad absoluta de nacimientos y la proporción de los nacimientos que son producto de madres adolescentes se ha mantenido estable debido a la inercia de crecimiento poblacional. ${ }^{3}$ Los nacimientos que ocurrieron entre mujeres menores de 20 años de edad representaron $17.1 \%$ del total de nacimientos en 1995 y contribuyeron con $13.6 \%$ de la Tasa General de Fecundidad. ${ }^{4,5}$ Se ha documentado ampliamente que el embarazo en edades tempranas, especialmente antes de los 18 años, pone en riesgo la salud de la madre ${ }^{6-8}$ y que los hijos de madres adolescentes -en circunstancias de pobreza y desnutrición- presentan mayor probabilidad de enfermar comparados con los hijos de madres mayores de 20 años de edad en las mismas condiciones sociales. ${ }^{9,10}$

Se sabe, además, que hay una asociación estadística entre maternidad temprana y baja escolaridad. ${ }^{11}$ Se ha señalado que "en la fecundidad total el peso relativo de los nacimientos de madres jóvenes es más elevado en los grupos de menor escolaridad y en las áreas rurales". ${ }^{12}$ Se ha argumentado que el embarazo en la etapa escolar las hace proclives a abandonar la escuela, aunque también se ha demostrado que es el abandono de la escuela por situaciones económicas lo que condiciona la maternidad precoz. ${ }^{13-15}$ Además, el embarazo cuando no es deseado representa mayor riesgo social, tanto para la madre como para la progenie. ${ }^{16,17}$

Se documentó en México, alrededor de los años 90, que el inicio de vida sexual ocurría en promedio -entre los hombres-a los 16 años y -entre las mujeresa los 19 años. ${ }^{18}$ Se ha observado que mientras la edad de inicio de la vida sexual activa se registra a edades más tempranas, la edad del matrimonio se ha postergado. De esta manera el lapso de fecundidad no marital se ha incrementado. 12,18,19

La Encuesta sobre el Comportamiento Reproductivo de los Adolescentes y Jóvenes del Area Metropolitana de la Ciudad de México (Ecram) realizada en México en 1988, entre población de 10 y 24 años de edad reveló que los jóvenes menores de 24 años después del inicio de la actividad sexual continúan activos y sin protección durante un año o más antes de solicitar un método anticonceptivo. Entre las mujeres sólo 36\% manifestó haber utilizado alguna práctica anticonceptiva en su primera relación sexual, ${ }^{18,19}$ y, entre las mujeres menores de 24 años que ya se habían embarazado alguna vez, más de 50\% manifestó que el primer embarazo no fue deseado. ${ }^{19}$

El objetivo de este trabajo fue documentar la proporción de nacimientos producto de madres adolescen- 
tes y describir la asociación de ciertas características demográficas con la manifestación sobre haber deseado el embarazo, así como con la utilización de métodos de anticoncepción posparto, en dos municipios del estado de Morelos, México.

\section{Material y métodos}

Este artículo describe los resultados de un estudio transversal con una muestra de 220 adolescentes entre 13 y 19 años de edad. El estudio original se hizo para evaluar una intervención de un modelo integral de atención a la mujer, concebido bajo un diseño cuasi experimental. Mediante un barrido censal en los municipios de Yecapixtla y Tlaltizapán, estado de Morelos, se registraron todos los hogares donde había mujeres entre 10 y 50 años de edad. Posteriormente, en dos mediciones hechas una en 1992, y otra en 1994, se registró a todas las mujeres embarazas $(n=1797)$. Para este análisis se excluyó al grupo de mujeres que recibió la intervención $(n=449)$, así como 32 registros, por corresponder a un segundo embarazo de una misma mujer en un periodo de estudio. Otros 58 registros se eliminaron por no tener la información completa, quedando 1258 mujeres. En este análisis se incluyó a las 220 mujeres que al momento de iniciar el embarazo tenían entre 13 y 19 años de edad, es decir $17.48 \%$ del total. Los criterios de inclusión fueron que la mujer manifestara estar embarazada, tuviera menos de seis meses de embarazo, y firmara consentimiento para participar en el estudio, además de residir en el municipio.

Una vez que el embarazo se detectó mediante un censo domiciliario, se pidió consentimiento para programar una segunda visita durante el segundo mes posterior a la fecha probable de parto. Mediante entrevista con la madre, se aplicó un cuestionario para evaluar la atención prenatal, la atención del parto y la planificación familiar posparto. La información se recolectó entre la sexta y décima segunda semana posparto. El cuestionario incluyó preguntas sobre composición familiar, escolaridad, indicadores previamente utilizados para conocer el estado socioeconómico, ${ }^{20}$ empleo asalariado, derechohabiencia, utilización de servicios médicos y de parteras, síntomas y complicaciones del embarazo y resultados perinatales. La sección de preguntas para evaluar la planificación familiar posparto inició con una para indagar sobre el deseo de tener un hijo antes del último embarazo. La pregunta se hizo de la siguiente manera: " $¿ a n t e s$ de este último embarazo, quería usted tener un hijo o hija, o no quería tener ningún(a) otro(a)?" Puesto que en una sección anterior del cuestionario se indagó si este era el primer embarazo, al tratarse de primíparas, la pregunta de la entrevistadora fue: " $i$ antes de este embarazo quería usted tener un hijo o hija?" Según la respuesta dicotómica -"sí" o "no"- se categorizó la población en dos grupos comparativos. El no-deseo constituyó la variable independiente del estudio. Las variables dependientes se definen en el cuadro I. Como variables de exposición se estudiaron características sociodemográficas, zona de residencia rural o urbana según la definición del Instituto Nacional de Estadística, Geografía e Informática $\left(\right.$ INEGI) ${ }^{21}$ e historia reproductiva. Además, se analizó el conocimiento y uso de métodos de planificación familiar posparto, según deseo del último embarazo. Entre las variables reproductivas, para este análisis, se consideró paridad, e intervalo intergenésico (cuadro I).

Para el análisis de la información se obtuvieron frecuencias simples sobre no deseo de embarazo, conocimiento de métodos anticonceptivos, y uso de método anticonceptivo al momento de la entrevista. Se calcularon las razones de momios crudas, e intervalos de confianza al 95\% del "no deseo de embarazo" de acuerdo con las variables independientes del estudio. Posteriormente se hizo un análisis multivariado mediante regresión logística no condicional, para ajustar por el posible efecto confusor de las variables incluidas en el modelo (edad, unión, escolaridad, nivel socioeconómico, derechohabiencia, zona de residencia y paridad). Finalmente, sobre el uso de métodos anticonceptivos posparto, se calcularon las razones de momios crudas e intervalos de confianza al 95\% de acuerdo con el no deseo del embarazo. Se utilizó, para la captura, el programa de D-Base 1995 y para el procesamiento y análisis el SPSS- W9.

\section{Resultados}

De las 220 adolescentes, $22.73 \%(n=50)$ manifestaron que no desearon el embarazo. Las características sociodemográficas y antecedentes reproductivos se muestran en el cuadro II. El 91.8\%, al momento de la aplicación de la entrevista (posparto) manifestaron estar unidas o casadas. Un $51.4 \%$ terminó la primaria y no continuó con los estudios de secundaria, $48.6 \%$ estaba cursando niveles superiores, $29.6 \%$ quedó en la categoría de estrato socioeconómico bajo, $69.1 \%$ estaba en la categoría de estrato socioeconómico medio y $47.3 \%$ habitaba en zona rural. De las variables de historia reproductiva encontramos que $68.2 \%$ manifestó que este era su primer embarazo, mientras que $31.8 \%$ cursaba su segundo embarazo o más. De las 70 que cursaban su segunda o tercera gesta se pudo obtener el intervalo intergenésico en 65 de ellas; $66.2 \%$ tuvo un 


\section{Cuadro I

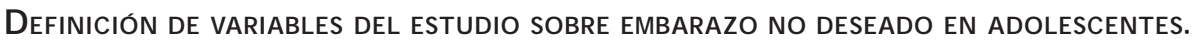 Morelos, MÉXICO, 1992-1994}

Adolescente

No deseo del embarazo

\begin{tabular}{ll} 
& primíparas: " ¿antes de este embarazo quería usted tener un hijo o hija?" \\
\hline Edad & $\begin{array}{l}\text { Edad de la mujer al momento de iniciar el embarazo } \\
\text { Se categorizó en dos grupos: } 13 \text { a } 17, \text { y } 18 \text { a } 19 \text { años de edad, ya que se ha documentado que el riesgo es aún mayor } \\
\text { antes de los } 18 \text { años }\end{array}$ \\
\hline Unión & $\begin{array}{l}\text { Unión: mujeres casadas y las mujeres con unión consensual } \\
\text { Sola: en esta categoría se clasificó a mujeres solteras al momento de la aplicación del cuestionario, viudas y divorciadas }\end{array}$
\end{tabular}

\begin{tabular}{ll}
\hline Escolaridad & $\begin{array}{l}\text { Se indagó sobre la escolaridad mediante dos preguntas. Una sobre el grado escolar alcanzado, y otra sobre el } \\
\text { número de años que asistió a la escuela }\end{array}$ \\
\hline Estrato socioeconómico & $\begin{array}{l}\text { Se utilizaron los indicadores del índice de Bronfmanne que incluye hacinamiento, condiciones de la vivienda, escolari- } \\
\text { dad del jefe de familia. Se consideró además la disponibilidad de electrodomésticos. Se estratificó en sólo dos } \\
\text { categorías: "alto" y "bajo" }\end{array}$ \\
\hline Derechohabiente & $\begin{array}{l}\text { Este término, en México, describe al individuo o población que por su filiación laboral está registrado en alguno de } \\
\text { los sistemas de seguridad social que incluye atención médica IMSS, ISSSTE, Pemex, Sedena) }\end{array}$ \\
\hline Zona & $\begin{array}{l}\text { Clasificación por A gregados Geo-Estadísticos Básicos (AG EBS) para el año 1995. Se define como rurales a las AG EBS } \\
\text { con menos de 2 } 500 \text { habitantes, y urbanas a las que tienen más habitantes (IN EG I) }\end{array}$ \\
\hline Intervalo intergenésico & $\begin{array}{l}\text { Se calculó el inter valo intergenésico con base en la fecha desenlace del penúltimo embarazo y la fecha del parto del } \\
\text { último. Se establecieron dos categorías: "menos de dos años" y "dos años o más" }\end{array}$ \\
\hline $\begin{array}{l}\text { Conocimiento de métodos } \\
\text { de planificación familiar }\end{array}$ & $\begin{array}{l}\text { Se le pidió a la encuestada que enunciara los métodos de planificación familiar que conocía. La entrevistadora } \\
\text { registró sus métodos mencionados }\end{array}$ \\
\hline $\begin{array}{l}\text { Métodos efectivos de planificación } \\
\text { familiar }\end{array}$ & $\begin{array}{l}\text { En esta categoría se agruparon "anticonceptivos hormonales orales e inyectables, el Dispositivo Intrauterino (DIU) } \\
\text { y al condón", aunque no hubo ni una en la última categoría }\end{array}$ \\
\hline
\end{tabular}

Uso de métodos anticonceptivos Se registró el tipo de método que se estaba utilizando al momento de la entrevista posparto

IMSS: Instituto Mexicano del Seguro Social

ISSSTE: Instituto de Seguridad y Servicios Sociales de los Trabajadores del Estado

Pemex: Petróleos Mexicanos

Sedena: Secretaría de D efensa N acional

intervalo de dos años o mayor, mientras que en $37.4 \%$ el intervalo fue menor de dos años.

En el cuadro III se muestran las razones de momios crudas y ajustadas de deseo de embarazo según las características sociodemográficas. Las razones de momios de no desear el embarazo fueron 2.42 veces mayores entre las mujeres no derechohabientes que entre las derechohabientes ( $R M=2.42$, IC 95\% 1.10, 5.32). Las razones de momios de no deseo fueron menores entre las mujeres primigestas que entre las multigestas $(R M=0.45$, IC $95 \% 0.23,0.86)$. No se encontró asociación cruda entre el no deseo de embarazo con la edad, unión, escolaridad, nivel socioeconómico, zona de residencia ni intervalo intergenésico. Se construyó un modelo de regresión logística no condicional multivariado, para estimar las razones de momios de no deseo de embarazo, incluyendo como variables predictoras edad, unión, escolaridad, nivel socioeconómico, derechohabiencia, zona de residencia y paridad. Al ajustar por todas estas variables en el modelo las razones de momios de no deseo de embarazo fueron 3.03 veces mayores entre las mujeres no derechohabientes que entre las derechohabientes $(R M=3.03$, IC 95\% 1.31, 7.00). Asimismo, las razones de momios de no desear el embarazo fueron dos veces más grandes entre mujeres de zona urbana que en las de zona rural ( $R M=2.16$, IC 95\% 1.08, 4.33). Las razones de momios de no desear el embarazo fueron 


\section{Cuadro II \\ Características sociodemográficas de la muestra PARA EL ESTUDIO SOBRE EMBARAZO NO DESEADO en Adolescentes. Morelos, México, 1992-1994}

\begin{tabular}{lrc} 
Variable & $n$ & $\%$ \\
Edad & & \\
13 a 17 años & 80 & 36.4 \\
\hline 18 a 19 años & 140 & 63.6 \\
Unión & & \\
$\quad$ Sola & 18 & 8.2 \\
\hline Unida & 202 & 91.8 \\
Escolaridad \\
$\quad$ Primaria \\
\hline Secundaria o más & & \\
\end{tabular}

N ivel socioeconómico

\begin{tabular}{lrl} 
Bajo & 155 & 70.4 \\
\hline Medio y alto & 65 & 29.6 \\
$\begin{array}{lrl}\text { Derechohabiente } \\
\text { Sí derechohabiente }\end{array}$ & \\
\hline No derechohabiente & 68 & 30.9 \\
\hline
\end{tabular}

Zona de residencia

\begin{tabular}{lll} 
Urbana & 116 & 52.7 \\
\hline Rural & 104 & 47.3
\end{tabular}

Paridad

\begin{tabular}{lrr} 
Primigesta & 70 & 31.8 \\
\hline 0 tro & 150 & 68.2
\end{tabular}

Intervalo intergenésico

(De 70 , se pudo calcular en 65)

\begin{tabular}{lll} 
Corto ( $<2$ años) & 22 & 37.4 \\
\hline 2 años o más & 43 & 66.2
\end{tabular}

$(n=220)$

menores entre las mujeres primigestas $(R M=0.43$, IC $95 \% 0.21,0.90)$. No se encontró asociación con las otras variables incluidas en el modelo (cuadro III).

Sobre el conocimiento y uso de métodos anticonceptivos (cuadro IV), 90.91\% enunció conocer los hormonales inyectables u orales como métodos de anticoncepción. Un 81.36\% mencionó el dispositivo intrauterino (DIU). Un 64.09\% enunció el condón. El $72.27 \%$ de las adolescentes atendieron su parto con un médico en centro de salud u hospital, mientras que
$27.73 \%$ lo atendieron en casa de la propia mujer o de la partera. Entre todas las adolescentes 59.54\% manifestó, después de seis semanas posparto, que no estaba utilizando ninguna práctica anticonceptiva. Un $4.54 \%$ manifestó que practicaba la lactancia o el coito interrumpido como método para no embarazarse, a pesar de saber que no son métodos efectivos. A tres adolescentes multíparas (1.36\%) les practicaron obturación tubaria bilateral (OTB). Un 10.45\% estaba utilizando hormonales, y $24.54 \%$ tenía el DIU. En suma, $34.99 \%$ utilizaba algún método efectivo. Sólo un poco más elevado fue el porcentaje entre aquellas a quienes les atendieron el parto en hospital o centro de salud. De $72.27 \%$ de las que lo tuvieron en un centro de salud u hospital $43.39 \%$ utilizaba algún método efectivo posparto (incluidas las tres con OTB). Finalmente, se calcularon las razones de momios de uso de métodos de planificación familiar según deseo de embarazo. Estos resultados se muestran en el cuadro V. No se encontró diferencia en los momios de utilización de métodos anticonceptivos en general, métodos efectivos, métodos hormonales o DIU, según deseo del embarazo.

El desconocimiento del DIU fue $45 \%$ mayor entre quienes no habían deseado el último embarazo ( $R M$ 1.45 IC 95\% 0.91, 2.31). No se encontró asociación significativa entre el deseo de embarazo y el conocimiento y uso de métodos de planificación familiar después del nacimiento.

\section{Discusión}

La proporción de nacimientos que son producto de madres adolescentes (17\%) es similar a la descrita para el estado de Morelos, ${ }^{22}$ y para todo el país en $1995 .^{3}$ Conviene destacar que casi dos terceras partes de las adolescentes embarazadas tenían 18 o 19 años y sólo un tercio era menor de 18 años. Esto es importante ya que el estereotipo de que la maternidad adolescente implica mayor riesgo obstétrico en realidad sólo se ha documentado en mujeres menores de 18 años, puesto que las mujeres entre 18 y 19 años de edad no muestran un riesgo mayor que las que tienen entre 20 y 24 años, ${ }^{24}$ excepto en condiciones donde además de la edad precoz hay situación de extrema pobreza., $10,23,24$ Más de una quinta parte de las adolescentes manifestó, en el posparto, no haber deseado un embarazo. No encontramos algún otro reporte epidemiológico sobre la frecuencia de embarazo adolescente no deseado en Morelos. La manifestación de no deseo del embarazo entre la población adolescente es menor a la que se reporta para la población general en la Encuesta Nacional de Fecundidad de $1987^{12}$ donde, hasta 50\% de las 


\section{Cuadro III \\ Prevalencias y razones de momios, Crudas y ajustadas,* de deseo del embarazo, de acuerdo con CaRACterísticas de las adolescentes de dos municipios. Morelos, México, 1999-1994}

\begin{tabular}{|c|c|c|c|c|c|c|}
\hline Variable & No desea & Sí desea & RM cruda & IC 95\% & RM ajustada & IC $95 \%$ \\
\hline \multicolumn{7}{|l|}{ Edad } \\
\hline 18 a 19 años & 36 & 104 & 0.61 & $0.31,1.22$ & 0.89 & 0.411 .93 \\
\hline 13 a 17 años & 14 & 66 & 1 & & & \\
\hline \multicolumn{7}{|l|}{ Unión } \\
\hline Sola & 4 & 14 & 0.97 & $(0.30,3.09)$ & 0.81 & 0.232 .78 \\
\hline Unida & 46 & 156 & 1 & & & \\
\hline \multicolumn{7}{|l|}{ Escolaridad } \\
\hline Primaria & 22 & 91 & 0.68 & $0.36,1.29$ & 0.52 & 0.261 .05 \\
\hline Secundaria 0 más & 28 & 79 & 1 & & & \\
\hline \multicolumn{7}{|l|}{ N ivel socioeconómico } \\
\hline 1 & 15 & 50 & 0.97 & $0.49,1.94$ & 0.92 & 0.431 .97 \\
\hline 0 & 35 & 120 & 1 & & & \\
\hline \multicolumn{7}{|l|}{ Derechohabiente } \\
\hline No derechohabiente & 41 & 111 & 2.42 & $01.10,5.32$ & 3.03 & 1.317 .00 \\
\hline Sí derechohabiente & 9 & 59 & 1 & & & \\
\hline \multicolumn{7}{|l|}{ Zona de residencia } \\
\hline Urbana & 18 & 86 & 1.82 & $0.95,3.49$ & 2.16 & 1.084 .33 \\
\hline Rural & 32 & 84 & 1 & & & \\
\hline \multicolumn{7}{|l|}{ Paridad } \\
\hline Primigesta & 23 & 47 & 0.45 & $0.23, .86$ & 0.43 & 0.210 .90 \\
\hline Otro & 27 & 123 & 1 & & & \\
\hline \multicolumn{7}{|c|}{ Intervalo intergenésico $(n=65)$} \\
\hline Corto (<2 años) & 8 & 14 & 1.07 & $(0.37,3.11)$ & & \\
\hline Largo (>2 años) & 15 & 28 & 1 & & & \\
\hline
\end{tabular}

*Ajustadas por edad, unión, escolaridad, nivel socioeconómico, derechohabiencia, zona de residencia y paridad

mujeres que habían tenido un hijo en los cinco años previos manifestó que no lo había planeado. En otro análisis, efectuado con la población original del estudio, se encontró que entre las mujeres de 20 a 34 años de edad la manifestación posparto de "no deseo del embarazo" fue de $33.51 \%$, y en mujeres de 35 y más años fue de $48.86 \%$.

El hallazgo sobre la mayor asociación entre no deseo del embarazo y ser no derechohabiente es consistente con reportes previos. ${ }^{12,19} \mathrm{El}$ hallazgo sobre la mayor asociación entre embarazo no deseado y residir en zona urbana rompe con el estereotipo que permeaba en la definición de la población objetivo de los programas del Consejo Nacional de Población, que señalaban a las mujeres rurales como población prioritaria en los programas de planificación familiar. ${ }^{12,23}$
Una limitación metodológica de este estudio es la dificultad para definir el deseo del embarazo. Esta definición ha sido ampliamente analizada por los doctores Feinholz ${ }^{25}$ y Vives. ${ }^{26}$ Además, la pregunta sobre el deseo del embarazo se hizo posparto. Feinholz y Avila han documentado la complejidad de la definición del "deseo de embarazo" y la evolución del deseo durante el embarazo. ${ }^{25}$ De un grupo de embarazadas, que al enterarse de su estado manifiestan verbalmente su rechazo por el mismo, una proporción considerable manifestará cambios de actitud a medida que éste progrese. Una vez concluido, la respuesta de la madre y del padre se ve influida no sólo por las experiencias agradables y desagradables del embarazo y del parto, sino, además, por la reciprocidad que exista entre las fantasías preconceptivas de los progenitores con las ca- 


\section{Cuadro IV \\ Conocimiento Y USO de MÉTOdos anticonceptivos ENTRE ADOLESCENTES CON EMBARAZO NO DESEADO. Morelos, México, 1992-1994}

\begin{tabular}{lrr}
$\begin{array}{l}\text { Conocimiento } \\
\text { Hormonales } \\
\text { Sí conoce }\end{array}$ & 200 & 90.91 \\
\hline $\begin{array}{l}\text { No conoce } \\
\text { Conoce DIU } \\
\text { Sí }\end{array}$ & 20 & 9.09 \\
\hline $\begin{aligned} \text { No } \\
\text { Conoce condón } \\
\text { Sí }\end{aligned}$ & 179 & 81.36 \\
\hline No & 41 & 18.64 \\
& 141 & 64.09 \\
\hline & 70 & 25.01
\end{tabular}

Práctica anticonceptiva

\begin{tabular}{lrr} 
N inguna & 131 & 59.54 \\
\hline Lactancia o coito interrumpido & 10 & 4.54 \\
\hline O bturación tubaria bilateral & 3 & 1.36 \\
\hline Hormonales & 23 & 10.45 \\
\hline Dispositivo intrauterino DIU & 54 & 24.54
\end{tabular}

Conoce algún método efectivo

\begin{tabular}{rrr} 
No & 141 & $64-09$ \\
\hline Sí & 79 & 34.54
\end{tabular}

Lugar de atención de parto y uso anticonceptivos

\begin{tabular}{lrr} 
A tención de parto hospitalario & 159 & 72.27 \\
\hline Usa método efectivo $(n=159)$ & 69 & 43.39 \\
\hline No usa método efectivo $(n=159)$ & 90 & 66.61
\end{tabular}

DIU: Dispositivo intrauterino

racterísticas reales de este particular bebé, como el sexo, el color de su piel, y el parecido con los padres, entre otras. ${ }^{26}$ Sobre la dimensión inconsciente del deseo de embarazarse, Vives y Lartigue han señalado que es necesario hacer la distinción entre el deseo de estar embarazada y el deseo de tener un hijo puesto que en la casuística clínica se reporta que el motivo de embarazarse es, en ocasiones, una respuesta a la necesidad de confirmar la capacidad reproductiva. ${ }^{26}$ Estos y otros autores han señalado que para algunas adolescentes el embarazarse representa la manera de salir de una situación familiar conflictiva. ${ }^{13,14,24,27}$ Vives argumenta, además, que todo deseo remite a la ausencia de lo anhelado y al reconocer la condición atemporal del deseo inconsciente, dice, una adolescente que piensa que tendrá un hijo en el futuro, de alguna manera tiene el anhelo en todo momento hasta que ese deseo queda satisfecho. ${ }^{25}$ Varios autores han argumentado que la manifestación sobre el deseo de un embarazo

\section{Cuadro $\mathrm{V}$ \\ Prevalencias y razones de Momios, conocimiento Y USO DE MÉTODOS DE PLANIFICACIÓN FAMILIAR, SEGÚN DESEO DEL EMBARAZO ENTRE ADOLESCENTES DE dos municipios de Morelos, México, 1992-1994}

Variable No desea Sí desea RM cruda IC 95\%

Conoce hormonales

\begin{tabular}{lrrll} 
No & 5 & 15 & 1.14 & $(025-0.79)$ \\
\hline Sí & 45 & 155 & 1 &
\end{tabular}

Conoce DIU

\begin{tabular}{lrrll} 
No & 4 & 37 & 0.31 & $0.10-0.92)$ \\
\hline Sí & 46 & 133 & 1 &
\end{tabular}

Conoce condón

\begin{tabular}{lrrll} 
No & 19 & 60 & 1.06 & $0.76-1.46$ \\
\hline Sí & 31 & 110 & 1 &
\end{tabular}

USA PF $(n=219)$

\begin{tabular}{lrrll} 
No & 30 & 106 & 0.95 & $0.50-1.83$ \\
\hline Sí & 19 & 64 & 1 &
\end{tabular}

Usa PF efectiva $(n=219)$

\begin{tabular}{lrrll} 
No & 32 & 111 & 1.00 & $0.51-1.95$ \\
\hline Sí & 17 & 59 & 1 &
\end{tabular}

Usa hormonal $(n=219)$

\begin{tabular}{lrrll} 
N o & 46 & 150 & 2.04 & $0.58-7.19$ \\
\hline Sí & 43 & 20 & 1 &
\end{tabular}

Usa DIU ( $n=219)$

\begin{tabular}{lrrll} 
No & 35 & 130 & 0.76 & $0.38-1.57$ \\
\hline Sí & 14 & 40 & 1 &
\end{tabular}

DIU: dispositivo intrauterino PF: planificación familiar

está determinada por factores socioculturales que varían dependiendo del contexto social, psicológico y cultural. ${ }^{24,27}$ Puesto que la información sobre la variable dependiente del estudio "no deseo del último embarazo" fue recolectada después del desenlace del embarazo, es posible que exista un sesgo de información, y las adolescentes informen con mayor frecuencia que sí deseaban el embarazo. Sin embargo, de acuerdo con toda la literatura revisada, este sesgo tendería a una subestimación de la frecuencia del no deseo ya que se ha documentado que entre un grupo de embarazadas la proporción de quienes manifiestan no desear el embarazo disminuye a medida que avanza el mismo y más todavía con el nacimiento del hijo o hija. Podemos inferir, entonces, que la proporción de mujeres que hubiesen manifestado que no desearon 
estar embarazadas sería mayor si se hubiese preguntado al inicio del embarazo, y aún mayor si se hubiese preguntado a la misma cohorte de mujeres antes de la concepción. ${ }^{25,28}$

La edad y la paridad se correlacionan positivamente. La manifestación de no deseo del embarazo se correlaciona con la multiparidad. Como se mencionó antes, entre las mujeres de 20 a 34 años de edad, la manifestación posparto de "no deseo del embarazo" fue de $33.51 \%$, y en mujeres de 35 y más años fue de $48.86 \%$. Figueroa ha argumentado que las respuestas que se obtienen de las madres en las encuestas que indagan sobre el deseo del último hijo, aluden más a la satisfacción de la pareja con el tamaño de familia deseada, es decir, al "número ideal de hijos" que la pareja había planeado, que al deseo o rechazo deliberado del último hijo. ${ }^{28}$

Hasta hace una década, en la literatura sobre fecundidad y planificación familiar solía asociarse la población rural con un mayor riesgo de exceso de fecundidad no planeada. ${ }^{29}$ Sin embargo, estudios más recientes realizados en zonas urbanas en América Latina documentan una mayor ocurrencia de embarazo no deseado en las zonas periféricas de las grandes urbes, sobre todo, en aquellas de inmigración acelerada $\mathrm{y}$ asentamientos marginados periurbanos. ${ }^{30} \mathrm{El}$ que se encuentre mayor asociación entre la manifestación de no deseo del embarazo con el hecho de residir en zona urbana, tiene que interpretarse con cautela. De acuerdo con los autores previamente citados, no sólo el deseo del embarazo está culturalmente determinado, sino también la factibilidad de que se manifieste que no se deseó un embarazo. ${ }^{14,15,24}$ De acuerdo con Román, en el contexto urbano la expectativa cultural es que la mujer joven continúe estudiando y posponga la crianza hasta después de los 24 años. ${ }^{14}$ En ese contexto es menos disonante que una mujer manifieste que no desea o deseó un embarazo, mientras que en una comunidad rural "las mujeres quieren tener hijos tempranamente en el ciclo vital", ya que tienen pocas opciones de "ser alguien" fuera de la maternidad. En estas circunstancias un embarazo, aunque no haya sido deliberadamente planeado, tiene más posibilidad de ser aceptado. Además, en ese contexto cultural es menos factible que, una vez nacido el bebé, se manifieste que el embarazo no fue deseado. ${ }^{13,25}$ Por otro lado, la menor asociación de embarazo no deseado en comunidades rurales podría sugerir que las acciones de las promotoras de planificación familiar han logrado coberturas eficaces. Se observa en las pequeñas comunidades rurales del estado de Morelos que las promotoras conocen a todas las mujeres potencialmente usuarias, y que deliberadamente programan sus visitas domiciliarias periódicas para buscarlas y ofrecerles métodos de anticoncepción. En contraste, en las zonas urbanas los servicios de planificación familiar tienen más un carácter de oferta abierta que de búsqueda proactiva e individual de posibles usuarias de métodos anticonceptivos. Además, en algunas zonas periurbanas la velocidad con que crecen los asentamientos humanos ponen un reto a los programas de servicios de salud que han sido incapaces de incrementar su cobertura a la misma velocidad.

Sobre los riesgos psicosociales que en la literatura se asocian con el maternaje adolescente, tanto Stern como otros autores previamente citados señalan que son las condiciones del contexto económico y cultural las que determinan tanto los significados de la sexualidad adolescente como el grado de aceptación de la familia y comunidad con la madre adolescente y su progenie. En una familia extensiva, donde se espera que la mujer se case y colabore en el cuidado de los hijos menores y con los quehaceres domésticos, el hecho de que una hija adolescente se embarace, o que un hijo traiga a casa a su mujer adolescente, es probable que encuentre aceptación por parte de otras mujeres adultas, así como mayor disponibilidad para cuidarle a ella y a su hijo. En contraste, en zonas urbanas donde predominan las familias nucleares, y donde la expectativa es que la mujer joven continúe estudiando y postergue la crianza, el embarazo adolescente representa un problema y es frecuentemente rechazado por la propia embarazada, el progenitor y las familias de ambos. $13,14,16,24,26,30$

En este estudio no pudo demostrarse -entre las adolescentes- la tan citada asociación estadística entre embarazo no deseado y escolaridad baja, ya que prácticamente todas las adolescentes manifestaron haber terminado la primaria o estarla concluyendo. Sin embargo, en otro análisis con la población original del estudio, se encontró que entre las mujeres mayores de 34 años hay una asociación significativa entre escolaridad menor de tres años y la frecuencia de embarazo no deseado entre las madres adolescentes. La baja escolaridad en México ha sido un indicador que resume situaciones de pobreza y de falta de acceso a los servicios de salud. Se argumenta que el embarazo hace proclive a la mujer a abandonar la escuela, sin embargo Stern sostiene que es el abandono temprano de la escuela y otras condiciones asociadas con la pobreza lo que deja a la mujer adolescente ante pocas alternativas de desarrollo, y que eso es lo que la hace proclive a la maternidad temprana. ${ }^{27,31}$ Menos de la mitad (48.6\%) había continuado con estudios secundarios o equivalentes. Conforme la cobertura que alcanzan las escuelas primarias en el territorio mexicano se ha extendido 
y la mayoría de la población va alcanzando niveles más altos de escolaridad, el umbral entre lo que constituye una baja y una alta escolaridad cambia, y posiblemente se esté desplazando hacia considerar como baja escolaridad a la secundaria inconclusa.

El estado conyugal y su asociación con el embarazo no deseado es difícil de explorar en una encuesta posparto, ya que se ha documentado que entre las adolescentes que se embarazan estando solteras, una proporción considerable se casa en el transcurso del embarazo. ${ }^{9}, 13,14,30$ Es oportuno, sin embargo, destacar una paradoja conceptual y política que todavía permea en las políticas de planificación familiar en México, y es que los programas de planificación familiar desarrollados por el Consejo Nacional de Población y por la Secretaría de Salud en México tenían como población objetivo a las mujeres unidas ${ }^{23} \mathrm{y}$, antes del advenimiento de los anticonceptivos no solía indagarse, entre mujeres casadas sobre su deseo del embarazo. Al mismo tiempo se asumía que las madres solteras tenían embarazos no intencionados debido a que "concibieron antes del matrimonio" (sic) ${ }^{27}$ En contraste, la Encuesta Nacional sobre Fecundidad realizada en 1987 reportó que hasta 50\% de las mujeres que habían procreado un hijo en los últimos cinco años, la mayoría de ellas casadas, informaron que el último no fue planeado. ${ }^{12}$ Estos hechos señalan la coexistencia paradójica de dos prejuicios sociales: que el embarazo no deseado sólo le ocurre a las mujeres solteras y, que, a pesar de ello, la promoción de los programas de planificación familiar debiera dirigirse a las mujeres unidas. La realidad es que mientras la edad promedio para contraer matrimonio se ha ido posponiendo, el inicio de la vida sexual se registra a edades más tempranas, por lo que la fracción de nacimientos atribuibles a concepción premarital ha aumentado. La proporción de nacimientos cuya concepción fue premarital aumentó de $10.5 \%$ en las cohortes de mujeres nacidas antes de 1949, a 16\% en la cohorte nacida entre 1960 y 1964. Mientras que en la generación más añosa el primogénito fue resultado de una concepción premarital, en una de cada 10 mujeres, en la cohorte de mujeres más jóvenes, nacidas entre 1960 y 1964, el primogénito de una de cada seis fue producto de una relación premarital., ${ }^{4}$ Es evidente la necesidad impostergable de priorizar en los programas de planificación familiar a las mujeres jóvenes, tanto solas como unidas.

El no ser derechohabiente, es decir, el no tener acceso al servicio médico de las instituciones públicas de seguridad social, Instituto Mexicano del Seguro Social, Instituto de Seguridad y Servicios Sociales de los Trabajadores del Estado, muestra correlación con la manifestación de no deseo del embarazo. Este dato es congruente con el reporte de uso de métodos de planificación en el posparto que es más alta entre quienes tuvieron acceso a la atención del parto en hospital, clínica o centro de salud. En este estudio el conocimiento se indagó de manera somera, es decir, se le pidió a la mujer que enunciara los métodos que conocía sin explorarse si conocían la manera de usarlos o dónde adquirirlos. El conocimiento de la existencia de métodos anticonceptivos, que expresaron la mayoría de las mamás adolescentes, al lado de una utilización tan baja en el posparto, sugiere que hay una brecha no bien elucidada entre tener el conocimiento y tener acceso a la adquisición de éste. No encontramos diferencias significativas en las razones de momios de no deseo del embarazo por estrato socioeconómico. Es sorprendente que tan sólo $45 \%$ de las jóvenes que se atendieron de parto en hospitales estaba utilizando algún método efectivo a seis semanas del posparto a pesar del consenso médico sobre la necesidad de alargar el intervalo intergenésico, especialmente en la población adolescente. ${ }^{6,7}$

Por otro lado, la brecha entre el conocimiento y el uso de métodos anticonceptivos, además de estar mediado por el costo y disponibilidad de los mismos, es posible que tenga relación con la perspectiva del deseo inconsciente del embarazo aportada por Vives. Siguiendo sus reflexiones, para controlar eficazmente la fecundidad un individuo necesita primero tomar conciencia de que es susceptible de embarazar o quedar embarazada. ${ }^{26}$ En este sentido los mensajes que promueven la postergación de la sexualidad y la abstinencia, pueden tener un efecto contrario al buscado, ya que hacen que el sujeto inconscientemente sienta que es posible dejar de ser susceptible de embarazarse, y por tanto, no decida deliberadamente el controlar su fecundidad mediante el uso de algún método anticonceptivo. Asimismo, las estrategias que promueven en el individuo la toma de conciencia de que se es susceptible de embarazarse propician en ellos la planeación deliberada de la fecundidad postergada. De ahí la importancia del cambio de enfoque propuesto por Stern y García, ${ }^{27,31}$ y la estrategia operacionalizada por Pick y Aguilar llamada "Planeando tu vida",,32 misma que ya se ha utilizado en algunas instituciones públicas como el Sistema Nacional para el Cuidado Integral de la Familia (DIF) en algunos estados, y en algunas organizaciones civiles.

En 35\% de las adolescentes que sí está utilizando algún método, el más utilizado fue el DIU. Esto refleja la influencia del programa de planificación familiar en los hospitales, donde el personal médico promueve la utilización del DIU como método anticonceptivo pos- 
parto, para espaciar el intervalo intergenésico sobre todo en mujeres jóvenes que aún planean tener más hijos en el futuro.

La discusión sobre las consecuencias que tiene en el desarrollo de un niño el ser producto de un embarazo que no fue deseado -aunque es un tema de suma trascendencia- está más allá del alcance de este artículo. Estudios longitudinales realizados en otros países que han comparado grupos de individuos que fueron producto de embarazos en los que se documentó el rechazo de la madre, con grupos en todo similares, excepto por el antecedente de rechazo del embarazo, reportan en la cohorte de los hijos una mayor incidencia de perturbaciones en el desarrollo, bajo desempeño y deserción escolar, drogadicción, delincuencia juvenil y divorcio, entre otros indicadores. ${ }^{25} \mathrm{El}$ deseo de los padres por el hijo es un determinante de estructuración psíquica del mismo, de su grado de autoestima, y de su capacidad amorosa, por eso, autores como Vives y Figueroa han propuesto que: "toda gestación en nuestras sociedades occidentales debería ser la consecuencia del deseo y la voluntad de los padres de tener un hijo." ${ }^{\prime 33}$

\section{Conclusiones}

Más de una quinta parte de los niños que nacen de madres adolescentes son producto de embarazos no deseados. La cobertura de métodos de anticoncepción posparto, aun entre adolescentes que se atendieron en centros médicos, no rebasa $45 \%$. Estos hallazgos apoyan las recomendaciones hechas con anterioridad en el sentido de que es "fundamental reducir el número de embarazos no planeados, no deseados, que terminan en abortos peligrosos o en niños que no son plenamente aceptados, a través de ofrecer alternativas accesibles para las mujeres de distintos contextos sociales". ${ }^{2}$ Las estrategias educativas en las que se promueve la deliberada planeación de una fecundidad postergada ${ }^{32}$ son intervenciones que prometen mayor efectividad que las desplegadas por los programas que promueven la abstinencia sexual o la postergación de su inicio. Coincidimos con otros autores en la necesidad de investigar más sobre los determinantes y las repercusiones del embarazo adolescente, y de evaluar la factibilidad de introducir en el programa escolar desde los últimos grados de la primaria, métodos de educación sexual que preparen al adolescente para la toma de conciencia de su capacidad para ejercer su sexualidad y planear deliberadamente la postergación de su fecundidad.

\section{Agradecimientos}

El estudio original se hizo bajo la conducción de la Dra. Ana Langer, en el Instituto Nacional de Salud Pública.

\section{Referencias}

1. Secretaría de Salud. Funciones prioritarias en salud. Revisión del Plan N acional de Salud. México, D.F.: SSA, 1997.

2. Langer A, Romero M. El embarazo, el parto y el puerperio. ¿Bajo qué condiciones se reproducen las mujeres en México? En: Langer A, Tolbert K, ed. México, D.F.:The Population Council, 1996:13-39.

3. Consejo Nacional de Población. La situación demográfica de México. México, D.F.: Ediciones de Consejo N acional de Población,1997:16.

4.Consejo N acional de Población. Programa N acional de Población 19952000. México, D.F.: Ediciones de Conapo, 1995:113.

5. Banco $\mathrm{N}$ acional de México. División de Estudios Económicos y Sociales. México Social- México, D.F.: Banamex, 1998:88-90.

6. Casanueva E, Soberanis T, O rtis, Bobadilla MA. Cambios en la composición corporal en el periodo perinatal en un grupo de adolescentes. Perinatol Reprod Hum 1991;5(1):28-32.

7. Moore S, Rosenthal D. Sexuality in adolescence. Ed. Routledge Adolescence Society Series, Londres: N ueva York 1993:23-43.

8. Schlaepfer L, Infante C. Patrones de inicio de la vida reproductiva: su relación con la mortalidad infantil y comportamientos reproductivos futuros. En: Lartigue T, Avila H, ed. Sexualidad y reproducción humana en México. México, D.F.: UIA-Plaza y Valdes, 1996; vol. (2):77-94.

9. Buvinic M, Valenzuela JP, Molina T, González E. La suerte de las madres adolescentes y sus hijos:la transmisión de la pobreza en Santiago de Chile. En: Schmukler B, comp. Familias y relaciones de género en transformación. México, D.F.: Population Council, Edamex, 1998:451-492.

10. Esco bedo E, Fletes J,VelázquezV. Embarazo en adolescentes: seguimiento de sus hijos durante el primer año de vida. Bol Med Hosp Infant Mex 1995;52(7):415-419.

11. Consejo Nacional de Población. Plan Nacional de Población. México, D.F.: Conapo, 1976.

12. Secretaría de Salud. El entorno de la regulación de la fecundidad en México. Serie: resultados de investigación. México, D.F.: SSA, 1993:189215.

13. Stern C. Embarazo adolescente; significados e implicaciones para distintos grupos sociales. D emos. Carta demográfica de México 1995(8).

14. Román R. Del primer vals al primer bebé: vivencias del embarazo en las jóvenes. Sonora, México, Instituto Mexicano de la Juventud -SEP, 2000;14:185-203.

15. Pick S, Atkin L, G ribble, Andrade-Palos. Sex contraception and pregnancy among adolescents in Mexico City. Stud Fam Plann 1991;22(2):7482.

16. Lartigue T, Vives J, Córdova A.A dolescentes y adultas embarazadas; un estudio psicológico comparativo. México, D.F.: U mbral XXI 1993;13:1620.

17. Vives J, Largitue T. Factores psicológicos del periodo perinatal: bases teóricas. En: Lartigue T, ed. Salud Comunitaria: una visión panamericana. México, D.F.: U niversidad Iberoamericana, 1991.

18.G arcía-Baltazar J, Figueroa-Perea JG. Práctica anticonceptiva en adolescentes y jóvenes del área metropolitana de la Ciudad de México. Salud Publica Mex 1992;34:(4):413-426. 
19. Secretaría de Salud. Encuesta sobre el Comportamiento Reproductivo de los Adolescentes y Jóvenes del A rea metropolitana de la Ciudad de México (ECRAMM 1988). México, D.F.: Dirección General de Planificación Familiar, 1989.

20. Bronfman M, Guiscafré H, C astro V, Castro R, G utiérrez G. La metodología y análisis de las características socio-económicas de la muestra. Arch Invest Med 1988;19:351-360.

21. Instituto Nacional de Estadística, Geografía e Informática. Listado de Agregados Geográficos de Estadística Básica del Estado de Morelos y municipios. México, D.F.: IN EGI, 1995.

22. Consejo Estatal de Población - Morelos. Breviario Demográfico de la Mujer en Morelos, México, 1998.

23. Consejo $\mathrm{N}$ acional de Población. Programa N acional de Educación Sexual 1976. México, D.F.: Conapo, 1976.

24.Villaseñor M,Alfaro N . Factores socioculturales que intervienen en la determinación del deseo o no deseo del embarazo en la adolescente. En: Lartigue T, Avila H, ed. Sexualidad y reproducción humana en México. México, D.F.: UIA-PlazaValdés, 1996; C apítulo IV:143-162.

25. Feinholz D,Avila H. Embarazo no deseado: el problema de la temporalidad En: Lartigue T, Avila H, ed. Sexualidad y reproducción humana en México. México, D.F.: UIA-PlazaValdés, 1996; C apítulo IV:113-142.
26.Vives J. El deseo de tener un hijo. En: Lartigue T,Avila H, ed. Sexualidad y reproducción humana en México, D.F.: UIA-Plaza Valdés, México 1996; Capítulo Il:99-112.

27. Stern C, García E. Hacia un nuevo enfoque en el campo del embarazo adolescente. Reflexiones 1999;2(13):1-99.

28. Figueroa JG. Preferencias reproductivas y posibilidades de interacción con programas y políticas de salud reproductiva. En: Lartigue T, Avila H, comp. Sexualidad y reproducción humana en México. México, D.F.: UIAPlaza Valdés, 1996;49-77.

29. O rganización Panamericana de la Salud. La salud de los adolescentes y jóvenes en las A méricas: un compromiso con el futuro.W ashington, D.C.: O rganización Panamericana de la Salud, 1985.

30. Eggleston E. D eterminants of unintended pregnancy among women in Ecuador. Int Perspect Fam Plann 1999;25:1-4.

31. Stern C. Prioridades de investigación para la prevención del embarazo adolescente en México: un punto de vista heterodoxo. Bol Salud Reprod Soc 1994;1(2):3-5.

32. Pick S,A guilar J. Planeando tu vida. Programa de educación sexual para adolescentes. México, D.F.: Ed. Pax, 1984.

33..Vives J, Lartigue T, comp.A pego y vínculo materno-infantil. Guadalajara, Jalisco: Universidad de Guadalajara, Jalisco, México, 1994:52-95 